\title{
Implicit Surface Reconstruction from Scattered Point Data with Noise
}

\author{
Jun Yang ${ }^{1,2}$, Zhengning Wang ${ }^{1}$, Changqian Zhu ${ }^{1}$, and Qiang Peng ${ }^{1}$ \\ ${ }^{1}$ School of Information Science \& Technology Southwest Jiaotong \\ University, Chengdu, Sichuan 610031 China \\ ${ }^{2}$ School of Mechanical \& Electrical Engineering Lanzhou Jiaotong \\ University, Lanzhou, Gansu 730070 China \\ yangj@mail.lzjtu.cn, \{znwang, cqzhu, pqiang\}@home.swjtu.edu.cn
}

\begin{abstract}
This paper addresses the problem of reconstructing implicit function from point clouds with noise and outliers acquired with 3D scanners. We introduce a filtering operator based on mean shift scheme, which shift each point to local maximum of kernel density function, resulting in suppression of noise with different amplitudes and removal of outliers. The "clean" data points are then divided into subdomains using an adaptive octree subdivision method, and a local radial basis function is constructed at each octree leaf cell. Finally, we blend these local shape functions together with partition of unity to approximate the entire global domain. Numerical experiments demonstrate robust and high quality performance of the proposed method in processing a great variety of $3 \mathrm{D}$ reconstruction from point clouds containing noise and outliers.
\end{abstract}

Keywords: filtering, space subdivision, radial basis function, partition of unity.

\section{Introduction}

The interest for point-based surface has grown significantly in recent years in computer graphics community due to the development of 3D scanning technologies, or the riddance of connectivity management that greatly simplifies many algorithms and data structures. Implicit surfaces are an elegant representation to reconstruct 3D surfaces from point clouds without explicitly having to account for topology issues. However, when the point sets data generated from range scanners (or laser scanners) contain large noise, especially outliers, some established methods often fail to reconstruct surfaces or real objects.

There are two major classes of surface representations in computer graphics: parametric surfaces and implicit surfaces. A parametric surface [1,2] is usually given by a function $f(s, t)$ that maps some 2-dimensional (maybe non-planar) parameter domain $\Omega$ into 3-space while an implicit surface typically comes as the zero-level isosurface of a 3 -dimensional scalar field $f(x, y, z)$. Implicit surface models are popular since they can describe complex shapes with capabilities for surface and volume modeling and complex editing operations are easy to perform on such models. 
Moving least square (MLS) [3-6] and radial basis function (RBF) [7-15] are two popular 3D implicit surface reconstruction methods.

Recently, RBF attracts more attention in surface reconstruction. It is identified as one of most accurate and stable methods to solve scattered data interpolation problems. Using this technique, an implicit surface is constructed by calculating the weights of a set of radial basis functions such they interpolate the given data points. From the pioneering work $[7,8]$ to recent researches, such as compactly-supported $\operatorname{RBF}[9,10]$, fast $\operatorname{RBF}$ [11-13] and multi-scale $\operatorname{RBF}[14,15]$, the established algorithms can generate more and more faithful models of real objects in last twenty years, unfortunately, most of them are not feasible for the approximations of unorganized point clouds containing noise and outliers.

In this paper, we describe an implicit surface reconstruction algorithm for noise scattered point clouds with outliers. First, we define a smooth probability density kernel function reflecting the probability that a point $\mathbf{p}$ is a point on the surface $S$ sampled by a noisy point cloud. A filtering procedure based on mean shift is used to move the points along the gradient of the kernel functions to the maximum probability positions. Second, we reconstruct a surface representation of "clean" point sets implicitly based on a combination of two well-known methods, RBF and partition of unity (PoU). The filtered domain of discrete points is divided into many subdomians by an adaptively error-controlled octree subdivision, on which local shape functions are constructed by RBFs. We blend local solutions together using a weighting sum of local subdomains. As you will see, our algorithm is robust and high quality.

\section{Filtering}

\subsection{Covariance Analysis}

Before introducing our surface reconstruction algorithm, we describe how to perform eigenvalue decomposition of the covariance matrix based on the theory of principal component analysis (PCA) [24], through which the least-square fitting plane is defined to estimate the kernel-based density function.

Given the set of input points $\Omega=\left\{\mathbf{p}_{i}\right\}_{i[1, L]}, \mathbf{p}_{i} \in \mathrm{R}^{3}$, the weighted covariance matrix $\mathbf{C}$ for a sample point $\mathbf{p}_{i} \in \Omega$ is determined by

$$
\mathbf{C}=\sum_{j=1}^{L}\left(\mathbf{p}_{j}-\overline{\mathbf{p}}_{i}\right)\left(\mathbf{p}_{j}-\overline{\mathbf{p}}_{i}\right)^{\mathrm{T}} \cdot \Psi\left(\left\|\mathbf{p}_{j}-\mathbf{p}_{i}\right\| / h\right),
$$

where $\overline{\mathbf{p}}_{i}$ is the centroid of the neighborhood of $\mathbf{p}_{i}, \Psi$ is a monotonically decreasing weight function, and $h$ is the adaptive kernel size for the spatial sampling density. Consider the eigenvector problem

$$
\mathbf{C} \cdot \mathbf{e}_{l}=\lambda_{l} \cdot \mathbf{e}_{l} .
$$

Since $\mathbf{C}$ is symmetric and positive semi-define, all eigenvalues $\lambda_{l}$ are real-valued and the eigenvectors $\mathbf{e}_{l}$ form an orthogonal frame, corresponding to the principal components of the local neighborhood. 
Assuming $\lambda_{0} \leq \lambda_{1} \leq \lambda_{2}$, it follows that the least square fitting plane $H(\mathbf{p})$ : $\left(\mathbf{p}-\overline{\mathbf{p}}_{i}\right) \cdot \mathbf{e}_{0}=0$ through $\overline{\mathbf{p}}_{i}$ minimizes the sum of squared distances to the neighbors of $\mathbf{p}_{i}$. Thus $\mathbf{e}_{0}$ approximates the surface normal $\mathbf{n}_{i}$ at $\mathbf{p}_{i}$, i.e., $\mathbf{n}_{i}=\mathbf{e}_{0}$. In other words, $\mathbf{e}_{1}$ and $\mathbf{e}_{2}$ span the tangent plane at $\mathbf{p}_{i}$.

\subsection{Mean Shift Filtering}

Mean shift $[16,17]$ is one of the robust iterative algorithms in statistics. Using this algorithm, the samples are shifted to the most likely positions which are local maxima of kernel density function. It has been applied in many fields of image processing and visualization, such as tracing, image smoothing and filtering.

In this paper, we use a nonparametric kernel density estimation scheme to estimate an unknown density function $g(\mathbf{p})$ of input data. A smooth kernel density function $g(\mathbf{p})$ is defined to reflect the probability that a point $\mathbf{p} \epsilon \mathrm{R}^{3}$ is a point on the surface $S$ sampled by a noisy point cloud $\Omega$. Inspired by the previous work of Schall et al. [21], we measure the probability density function $g(\mathbf{p})$ by considering the squared distance of $\mathbf{p}$ to the plane $\mathrm{H}(\mathbf{p})$ fitted to a spatial $k$-neighborhood of $\mathbf{p}_{i}$ as

$$
g(\mathbf{p})=\sum_{i=1}^{L} g_{i}(\mathbf{p})=\sum_{i=1}^{L} \Phi_{i}\left(\mathbf{p}-\mathbf{p}_{\text {pro }}\right) G_{i}\left(\mathbf{p}_{\text {pro }}-\overline{\mathbf{p}}_{i}\right)\left\{1-\left[\left(\mathbf{p}-\overline{\mathbf{p}}_{i}\right) \cdot \mathbf{n}_{i} / h\right]^{2}\right\},
$$

where $\Phi_{i}$ and $\mathrm{G}_{i}$ are two monotonically decreasing weighting functions to measure the spatial distribution of point samples from spatial domain and range domain, which are more adaptive to the local geometry of the point model. The weight function could be either a Gaussian kernel or an Epanechnikov kernel. Here we choose Gaussian function $e^{-x^{2} / 2 \sigma^{2}}$. The $\mathbf{p}_{\text {pro }}$ is an orthogonal projection of a certain sample point $\mathbf{p}$ on the least-square fitting plane. The positions $\mathbf{p}$ close to $\mathrm{H}(\mathbf{p})$ will be assigned with a higher probability than the positions being more distant.

The simplest method to find the local maxima of (3) is to use a gradient-ascent process written as follows:

$$
\nabla g(\mathbf{p})=\sum_{i=1}^{L} \nabla g_{i}(\mathbf{p}) \approx \frac{-2}{h^{2}} \sum_{i=1}^{L} \Phi_{i}\left(\mathbf{p}-\mathbf{p}_{\mathrm{pro}}\right) G_{i}\left(\mathbf{p}_{\mathrm{pro}}-\overline{\mathbf{p}}_{i}\right)\left[\left(\mathbf{p}-\overline{\mathbf{p}}_{i}\right) \cdot \mathbf{n}_{i}\right] \cdot \mathbf{n}_{i} .
$$

Thus the mean shift vectors are determined as

$$
m(\mathbf{p})=\mathbf{p}-\left\{\sum_{i=1}^{L} \Phi_{i}\left(\mathbf{p}-\mathbf{p}_{\text {pro }}\right) G_{i}\left(\mathbf{p}_{\mathrm{pro}}-\overline{\mathbf{p}}_{i}\right)\left[\left(\mathbf{p}-\overline{\mathbf{p}}_{i}\right) \cdot \mathbf{n}_{i}\right] \cdot \mathbf{n}_{i} / \sum_{i=1}^{L} \Phi_{i}\left(\mathbf{p}-\mathbf{p}_{\mathrm{pro}}\right) G_{i}\left(\mathbf{p}_{\mathrm{pro}}-\overline{\mathbf{p}}_{i}\right)\right\} .
$$

Combining equations (4) and (5) we get the resulting iterative equations of mean shift filtering

$$
\mathbf{p}_{i}^{j+1}=m\left(\mathbf{p}_{i}^{j}\right), \quad \mathbf{p}_{i}^{o}=\mathbf{p}_{i},
$$

where $j$ is the number of iteration. In our algorithm, $g(\mathbf{p})$ satisfies the following conditions

$$
g\left(\mathbf{p}_{2}\right)-g\left(\mathbf{p}_{1}\right)>\nabla g\left(\mathbf{p}_{1}\right)\left(\mathbf{p}_{2}-\mathbf{p}_{1}\right) \quad \forall \mathbf{p}_{1} \geq 0, \forall \mathbf{p}_{2} \geq 0
$$


thus $g(\mathbf{p})$ is a convex function with finite stable points in the set $U=\left\{\mathbf{p}_{i} \mid g\left(\mathbf{p}_{i}\right) \geq g\left(\mathbf{p}_{i}^{1}\right)\right\}$ resulting in the convergence of the series $\left\{\mathbf{p}_{i}^{j}, i=1, \ldots, L, j=1,2, \ldots\right\}$. Experiments show that we stop iterative process if $\left\|\mathbf{p}_{i}^{j+1}-\mathbf{p}_{i}^{j}\right\| \leq 5 \times 10^{-3} h$ is satisfied. Each sample usually converges in less than 8 iterations. Due to the clustering property of our method, groups of outliers usually converge to a set of single points sparsely distributed around the surface samples. These points can be characterized by a very low spatial sampling density compared to the surface samples. We use this criteria for the detection of outliers and remove them using a simple threshold.

\section{Implicit Surface Reconstruction}

\subsection{Adaptive Space Subdivision}

In order to avoid solving a dense linear system, we subdivide the whole input points filtered by mean shift into slightly overlapping subdomains. An adaptive octree-based subdivision method introduced by Ohtake et al. [18] is used in our space partition.

We define the local support radius $R=\alpha d_{i}$ for the cubic cells which are generated during the subdivision, $d_{i}$ is the length of the main diagonal of the cell. Assume each cell should contain points between $\mathrm{T}_{\min }$ and $\mathrm{T}_{\max }$. In our implementation, $\alpha=0.6, \mathrm{~T}_{\min }$ $=20$ and $\mathrm{T}_{\max }=40$ has provided satisfying results.

A local max-norm approximation error is estimated according to the Taubin distance [19],

$$
\varepsilon=\max _{\left|\mathbf{p}_{i}-c_{i}\right|<R}\left|f\left(\mathbf{p}_{i}\right)\right| /\left|\nabla f\left(\mathbf{p}_{i}\right)\right| .
$$

If the $\varepsilon$ is greater than a user-specified threshold $\varepsilon_{0}$, the cell is subdivided and a local neighborhood function $f_{i}$ is built for each leaf cell.

\subsection{Estimating Local Shape Functions}

Given the set of $N$ pairwise distinct points $\Omega=\left\{\mathbf{p}_{i}\right\}_{i \in[1, N]}, \mathbf{p}_{i} \in \mathrm{R}^{3}$, which is filtered by mean shift algorithm, and the set of corresponding values $\left\{v_{i}\right\}_{i \in[1, N]}, v_{i} \in \mathrm{R}$, we want to find an interpolation $f: \mathrm{R}^{3} \rightarrow \mathrm{R}$ such that

$$
f\left(\mathbf{p}_{i}\right)=v_{i} .
$$

We choose the $f(\mathbf{p})$ to be a radial basis function of the form

$$
f(\mathbf{p})=\eta(\mathbf{p})+\sum_{i=1}^{N} \omega_{i} \varphi\left(\left|\mathbf{p}-\mathbf{p}_{i}\right|\right),
$$

where $\eta(\mathbf{p})=\zeta_{k} \eta_{k}(\mathbf{p})$ with $\left\{\eta_{k}(\mathbf{p})\right\}_{k \in[1, Q]}$ is a basis in the 3D null space containing all real-value polynomials in 3 variables and of order at most $m$ with $Q=\left\{\begin{array}{c}m+3 \\ 3\end{array}\right\}$ depending on the choice of $\varphi, \varphi$ is a basis function, $\omega_{i}$ are the weights in real numbers, and I. I denotes the Euclidean norm. 
There are many popular basis functions $\varphi$ for use: biharmonic $\varphi(r)=r$, triharmonic $\varphi(r)=r^{3}$, multiquadric $\varphi(r)=\left(r^{2}+c^{2}\right)^{1 / 2}$, Gaussian $\varphi(r)=\exp \left(-c r^{2}\right)$, and thin-plate spline $\varphi(r)=r^{2} \log (r)$, where $r=\left|\mathbf{p}-\mathbf{p}_{i}\right|$.

As we have an under-determined system with $N+Q$ unknowns and $N$ equations, socalled natural additional constraints for the coefficient $\omega_{i}$ are added in order to ensure orthogonality, so that

$$
\sum_{i=1}^{N} \omega_{i} \eta_{1}=\sum_{i=1}^{N} \omega_{i} \eta_{2}=\cdots=\sum_{i=1}^{N} \omega_{i} \eta_{Q}=0 .
$$

The equations (9), (10) and (11) may be written in matrix form as

$$
\left(\begin{array}{cc}
\mathbf{A} & \boldsymbol{\eta} \\
\boldsymbol{\eta}^{\mathbf{T}} & \mathbf{0}
\end{array}\right)\left(\begin{array}{l}
\boldsymbol{\omega} \\
\zeta
\end{array}\right)=\left(\begin{array}{l}
\mathbf{v} \\
0
\end{array}\right)
$$

where $\mathbf{A}=\varphi\left(\left|\mathbf{p}_{i}-\mathbf{p}_{j}\right|\right), i, j=1, \ldots, N, \boldsymbol{\eta}=\eta_{k}\left(\mathbf{p}_{i}\right), i=1, \ldots, N, k=1, \ldots, Q, \boldsymbol{\omega}=\omega_{i}, i=1, \ldots, N$ and $\zeta=\zeta_{k}, k=1, \ldots, Q$. Solving the linear system (14) determines $\omega_{i}$ and $\zeta_{k}$, hence the $f(\mathbf{p})$.

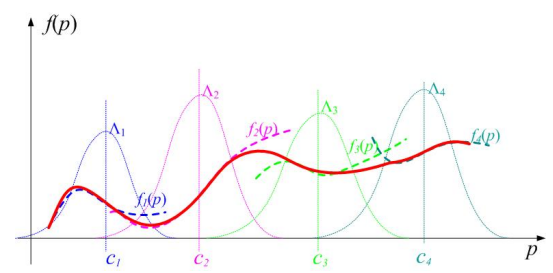

Fig. 1. A set of locally defined functions are blent by the PoU method. The resulting function (solid curve) is constructed from four local functions (thick dashed curves) with their associated weight functions (dashed dotted curves).

\subsection{Partition of Unity}

After suppressing high frequency noise and removing outliers, we divide the global domain $\Omega=\left\{\mathbf{p}_{i}\right\}_{i \in[1, N]}$ into $M$ lightly overlapping subdomains $\left\{\Omega_{i}\right\}_{i \in[1, M]}$ with $\Omega \subseteq \bigcup_{i} \Omega_{i}$ using an octree-based space partition method. On this set of subdomains $\left\{\Omega_{i}\right\}_{i \in[1, M]}$, we construct a partition of unity, i.e., a collection of non-negative functions $\left\{\Lambda_{i}\right\}_{i \in[1, M]}$ with limited support and with $\sum \Lambda_{i}=1$ in the entire domain $\Omega$. For each subdomain $\Omega_{i}$ we construct a local reconstruction function $f_{i}$ based on RBF to interpolate the sampled points. As illustrated in Fig. 1, four local functions $f_{l}(\mathbf{p}), f_{2}(\mathbf{p}), f_{3}(\mathbf{p})$ and $f_{4}(\mathbf{p})$ are blended together by weight functions $\Lambda_{1}, \Lambda_{2}, \Lambda_{3}$ and $\Lambda_{4}$. The solid curve is the final reconstructed function.

Now an approximation of a function $f(\mathbf{p})$ defined on $\Omega$ is given by a combination of the local functions

$$
f(\mathbf{p})=\sum_{i=1}^{M} f_{i}(\mathbf{p}) \Lambda_{i}(\mathbf{p}) .
$$

The blending function is obtained from any other set of smooth functions by a normalization procedure 


$$
\Lambda_{i}(\mathbf{p})=w_{i}(\mathbf{p}) / \sum_{j} w_{j}(\mathbf{p}) \cdot
$$

The weight functions $w_{i}$ must be continuous at the boundary of the subdomains $\Omega_{i}$. Tobor et al. [15] suggested that the weight functions $w_{i}$ be defined as the composition of a distance function $\mathrm{D}_{i}: \mathrm{R}^{\mathrm{n}} \rightarrow[0,1]$, where $\mathrm{D}_{i}(\mathbf{p})=1$ at the boundary of $\Omega_{i}$ and a decay function $\theta:[0,1] \rightarrow[0,1]$, i.e. $w_{i}(\mathbf{p})=\theta \circ \mathrm{D}_{i}(\mathbf{p})$. More details about $\mathrm{D}_{i}$ and $\theta$ can be found in Tobor's paper.

Table 1. Computational time measurements for mean shift filtering and RBF+PoU surface reconstructing with error bounded at $10^{-5}$. Timings are listed as minutes:seconds.

\begin{tabular}{|l|l|l|l|}
\hline model & Bunny & Dragon head & Dragon \\
\hline $\mathrm{P}_{\text {input }}$ & $362 \mathrm{~K}$ & $485 \mathrm{~K}$ & $2.11 \mathrm{M}$ \\
\hline $\mathrm{P}_{\text {filter }}$ & $165 \mathrm{~K}$ & $182 \mathrm{~K}$ & $784 \mathrm{~K}$ \\
\hline $\mathrm{T}_{\text {filter }}$ & $9: 07$ & $13: 26$ & $41: 17$ \\
\hline $\mathrm{T}_{\text {octree }}$ & $0: 02$ & $0: 04$ & $0: 10$ \\
\hline $\mathrm{T}_{\text {rec }}$ & $0: 39$ & $0: 51$ & $3: 42$ \\
\hline
\end{tabular}

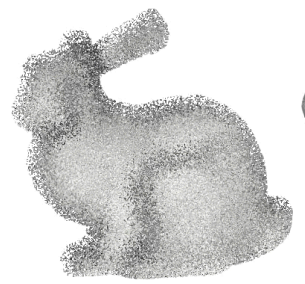

(a)

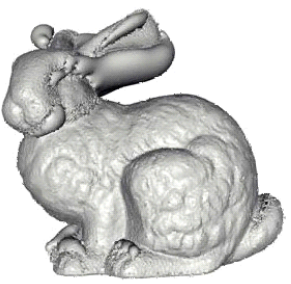

(b)

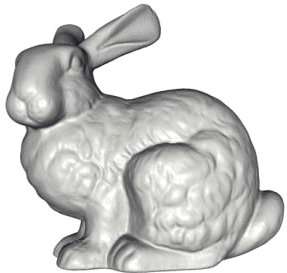

(c)

Fig. 2. Comparison of implicit surface reconstruction based on RBF methods. (a) Input noisy point set of Stanford bunny (362K). (b) Reconstruction with Carr's method [11]. (c) Reconstruction with our method in this paper.

\section{Applications and Results}

All results presented in this paper are performed on a $2.8 \mathrm{GHz}$ Intel Pentium4 PC with $512 \mathrm{M}$ of RAM running Windows XP.

To visualize the resulting implicit surfaces, we used a pure point-based surface rendering algorithm such as [22] instead of traditionally rendering the implicit surfaces using a Marching Cubes algorithm [23], which inherently introduces heavy topological constraints.

Table 1 presents computational time measurements for filtering and reconstructing of three scan models, bunny, dragon head and dragon, with user-specified error threshold $10^{-5}$ in this paper. In order to achieve good effects of denoising we choose a large number of $k$-neighborhood for the adaptive kernel computation, however, more timings of filtering are spent . In this paper, we set $k=200$. Note that the filtered points are less than input noisy points due to the clustering property of our method.

In Fig. 2 two visual examples of the reconstruction by Carr's method [11] and our algorithm are shown. Carr et al. use polyharmonic RBFs to reconstruct smooth, 
manifold surfaces from point cloud data and their work is considered as an excellent and successful research in this field. However, because of sensitivity to noise, the reconstructed model in the middle of Fig. 2 shows spurious surface sheets. The quality of the reconstruction is highly satisfactory, as be illustrated in the right of Fig. 2, since a mean shift operator is introduced to deal with noise in our algorithm.

For the purpose of illustrating the influence of error thresholds on reconstruction accuracy and smoothness, we set two different error thresholds on the reconstruction of the scanned dragon model, as demonstrated by Fig. 3 .

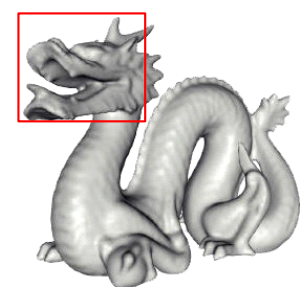

(a)

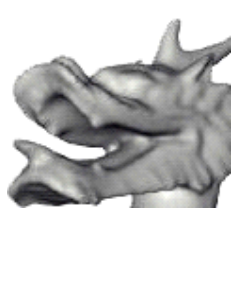

(b)

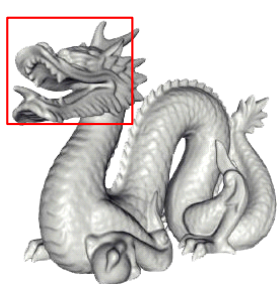

(c)

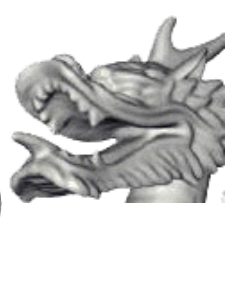

(d)

Fig. 3. Error threshold controls reconstruction accuracy and smoothness of the scanned dragon model consisting of $2.11 \mathrm{M}$ noisy points. (a) Reconstructing with error threshold at $8.4 \times 10^{-4}$. (c) Reconstructing with error threshold at $2.1 \times 10^{-5}$. (b) and (d) are close-ups of the rectangle areas of (a) and (c) respectively.

\section{Conclusion and Future Work}

In this study, we have presented a robust method for implicit surface reconstruction from scattered point clouds with noise and outliers. Mean shift method filters the raw scanned data and then the PoU scheme blends the local shape functions defined by RBF to approximate the whole surface of real objects.

We are also investigating various other directions of future work. First, we are trying to improve the space partition method. We think that the Volume-Surface Tree [20], an alternative hierarchical space subdivision scheme providing efficient and accurate surface-based hierarchical clustering via a combination of a global 3D decomposition at coarse subdivision levels, and a local 2D decomposition at fine levels near the surface may be useful. Second, we are planning to combine our method with some feature extraction procedures in order to adapt it for processing very incomplete data.

\section{References}

1. Weiss, V., Andor, L., Renner, G., Varady, T.: Advanced Surface Fitting Techniques. Computer Aided Geometric Design, 1 (2002) 19-42

2. Iglesias, A., Echevarría, G., Gálvez, A.: Functional Networks for B-spline Surface Reconstruction. Future Generation Computer Systems, 8 (2004) 1337-1353

3. Alexa, M., Behr, J., Cohen-Or, D., Fleishman, S., Levin D., Silva, C. T.: Point Set Surfaces. In: Proceedings of IEEE Visualization. San Diego, CA, USA, (2001) 21-28

4. Amenta, N., Kil, Y. J.: Defining Point-Set Surfaces. ACM Transactions on Graphics, 3 (2004) 264-270 
5. Levin, D.: Mesh-Independent Surface Interpolation. In: Geometric Modeling for Scientific Visualization, Spinger-Verlag, (2003) 37-49

6. Fleishman, S., Cohen-Or, D., Silva, C. T.: Robust Moving Least-Squares Fitting with Sharp Features. ACM Transactions on Graphics, 3 (2005) 544-552

7. Savchenko, V. V., Pasko, A., Okunev, O. G., Kunii, T. L.: Function Representation of Solids Reconstructed from Scattered Surface Points and Contours. Computer Graphics Forum, 4 (1995) 181-188

8. Turk, G., O’Brien, J.: Variational Implicit Surfaces. Technical Report GIT-GVU-99-15, Georgia Institute of Technology, (1998)

9. Wendland, H.: Piecewise Polynomial, Positive Definite and Compactly Supported Radial Functions of Minimal Degree. Advances in Computational Mathematics, (1995) 389-396

10. Morse, B. S., Yoo, T. S., Rheingans, P., Chen, D. T., Subramanian, K. R.: Interpolating Implicit Surfaces from Scattered Surface Data Using Compactly Supported Radial Basis Functions. In: Proceedings of Shape Modeling International, Genoa, Italy, (2001) 89-98

11. Carr, J. C., Beatson, R. K., Cherrie, J. B., Mitchell, T. J., Fright, W. R., McCallum, B. C., Evans, T. R.: Reconstruction and Representation of 3D Objects with Radial Basis Functions. In: Proceedings of ACM Siggraph 2001, Los Angeles, CA , USA, (2001) 67-76

12. Beatson, R. K.: Fast Evaluation of Radial Basis Functions: Methods for Two-Dimensional Polyharmonic Splines. IMA Journal of Numerical Analysis, 3 (1997) 343-372

13. Wu, X., Wang, M. Y., Xia, Q.: Implicit Fitting and Smoothing Using Radial Basis Functions with Partition of Unity. In: Proceedings of 9th International Computer-AidedDesign and Computer Graphics Conference, Hong Kong, China, (2005) 351-360

14. Ohtake, Y., Belyaev, A., Seidel, H. P.: Multi-scale Approach to 3D Scattered Data Interpolation with Compactly Supported Basis Functions. In: Proceedings of Shape Modeling International, Seoul, Korea, (2003) 153-161

15. Tobor, I., Reuter, P., Schlick, C.: Multi-scale Reconstruction of Implicit Surfaces with Attributes from Large Unorganized Point Sets. In: Proceedings of Shape Modeling International, Genova, Italy, (2004) 19-30

16. Comaniciu, D., Meer, P.: Mean Shift: A Robust Approach toward Feature Space Analysis. IEEE Transactions on Pattern Analysis and Machine Intelligence, 5 (2002) 603-619

17. Cheng, Y. Z.: Mean Shift, Mode Seeking, and Clustering. IEEE Transactions on Pattern Analysis and Machine Intelligence, 8 (1995) 790-799

18. Ohtake, Y., Belyaev, A., Alexa, M., Turk, G., Seidel, H. P.: Multi-level Partition of Unity Implicits. ACM Transactions on Graphics, 3 (2003) 463-470

19. Taubin, G.: Estimation of Planar Curves, Surfaces and Nonplanar Space Curves Defined by Implicit Equations, with Applications to Edge and Range Image Segmentation. IEEE Transaction on Pattern Analysis and Machine Intelligence, 11 (1991) 1115-1138

20. Boubekeur, T., Heidrich, W., Granier, X., Schlick, C.: Volume-Surface Trees. Computer Graphics Forum, 3 (2006) 399-406

21. Schall, O., Belyaev, A., Seidel, H-P.: Robust Filtering of Noisy Scattered Point Data. In: IEEE Symposium on Point-Based Graphics, Stony Brook, New York, USA, (2005) 71-77

22. Rusinkiewicz, S., Levoy, M.: Qsplat: A Multiresolution Point Rendering System for Large Meshes. In: Proceedings of ACM Siggraph 2000, New Orleans, Louisiana, USA, (2000) 343-352

23. Lorensen, W. E., Cline, H. F.: Marching Cubes: A High Resolution 3D Surface Construction Algorithm. Computer Graphics, 4 (1987) 163-169

24. Hoppe, H., DeRose, T., Duchamp, T., McDonald, J., Stuetzle, W.: Surface Reconstruction from Unorganized Points. In: Proceedings of ACM Siggraph'92, Chicago, Illinois, USA, (1992) 71-78 